\title{
Association of acanthosis nigricans with race and metabolic disturbances in obese women
}

L.M.B. Araújo ${ }^{1}$, M.V. Porto ${ }^{1}$, E.M. Netto ${ }^{2}$ and M.J. Ursich ${ }^{3}$

\section{Correspondence \\ L.M.B. Araújo \\ Seção de Endocrinologia \\ Hospital U niversitário \\ Prof. Edgard Santos \\ Rua Augusto Viana, $s / \mathrm{n}, 60$ andar \\ 40110-160 Salvador, BA \\ Brasil \\ Fax: + 55-71-247-8492 \\ E-mail: Imba@ufba.br}

L.M.B. Araújo is the recipient of a CNPq Scientific Productivity grant. Publication supported by FAPESP.

Received April 16, 2001 Accepted 0 ctober 16, 2001

\author{
${ }^{1}$ Seção de Endocrinologia, Hospital Universitário Prof. Edgard Santos, \\ Faculdade de Medicina, Universidade Federal da Bahia, Salvador, BA, Brasil \\ ${ }^{2}$ Fundação de Infectologia da Bahia, Universidade Federal da Bahia, \\ Salvador, BA, Brasil \\ ${ }^{3}$ Laboratório de Investigação M édica (LIM 18), Faculdade de Medicina, \\ Universidade de São Paulo, São Paulo, SP, Brasil
}

\section{Abstract}

Acanthosis nigricans ( $\mathrm{AN}$ ) has been recognized as a marker of insulin resistance and diabetes mellitus. We have compared frequency of race and metabolic disturbances in obese women with several degrees of AN (AN group, $N=190$ ) to a group without AN (non-AN group, $N=$ 61 ) from a mixed racial population. The groups were similar regarding age and body mass index. All patients (except the diabetic patients) underwent an oral glucose tolerance test $(75 \mathrm{~g})$. The racial distribution of this population was $35.1 \%$ white, $37.8 \%$ mulatto and $27.1 \%$ black and the frequency of AN was $62.5,82.1$ and $83.8 \%$, respectively, higher in black versus white $(\mathrm{P}=0.003)$ and mulatto versus white $(\mathrm{P}$ $=0.002$ ) women. The frequencies of diabetes mellitus and impaired glucose tolerance were 5.8 and $12.6 \%$ in the AN group and 1.6 and $8.2 \%$ in the non-AN group, respectively $(\mathrm{P}>0.05)$. Fasting glucose, $B$ cell function determined by the homeostasis model of assessment (HOMA), fasting insulin and insulin area under the curve were similar for the AN and non-AN groups. A higher HOMA insulin resistance was observed in the AN group compared to the non- $\mathrm{AN}$ group $(\mathrm{P}=$ 0.02 ) and in the subgroup of highest degree of AN compared to those with other degrees. The mean lipid levels and the frequency of dyslipidemia were similar for the two groups. AN was strongly associated with the black or mulatto rather than the white race, even after taking into account the effect of age, body mass index and HOMA insulin resistance.

\section{Introduction}

Acanthosis nigricans (AN) is a cutaneous abnormality characterized by a hyperpigmented velvety cutaneous thickening that can occur on any part of the body: axillae, nape and sides of the neck, the groin, antecubital and popliteal surfaces, umbilical area and even mucosal surfaces. Both hyperpig-

\author{
Key words \\ - Acanthosis nigricans \\ - Race \\ - O besity \\ - Insulin resistance \\ - Hyperinsulinism
}

..................... 
tance. Other metabolic conditions such as diabetes mellitus and impaired glucose tolerance and some other clusters of metabolic syndromes such as dyslipidemia, polycystic ovary and arterial hypertension could also be associated. Several other endocrine diseases are also associated with AN: acromegaly, hyperprolactinemia, Cushing's syndrome, hirsutism, hyperthyroidism, hypothyroidism, Addison's disease, and lipoatrophic diabetes (8). Some of these conditions are also associated with obesity and insulin resistance.

Several investigators have described the prevalence of AN in small groups of obese patients and some of them have shown increased fasting insulin levels in patients with AN compared to patients without AN $(8,10-12)$. Insulin resistance was evaluated in few reports. In a larger study of 137 Mexican Americans with AN, Burke et al. (16) suggested a quantitative scale of $A N$ and showed a strong association of this scale with fasting insulin and body mass index (BMI). They also described an association with elevated fasting glucose and low HDLcholesterol in non-diabetic patients and with elevated cholesterol levels only in diabetic subjects.

In the present investigation, we studied insulin secretion in response to glucose overload, insulin resistance and lipid profile in obese women from a mixed racial population with and without $\mathrm{AN}$ to determine if $\mathrm{AN}$ is associated with race, insulin resistance and higher frequency of metabolic disturbances.

\section{Material and Methods}

A total of 251 obese women (BMI from 30.3 to $71.9 \mathrm{~kg} / \mathrm{m}^{2}$ ) from an obesity outpatient clinic who were seeking obesity treatment were enrolled consecutively prior to being placed on a diet. All patients with chronic diseases or taking medication that would change glucose tolerance or induce
AN were excluded. This research was approved by the Prof. Edgard Santos Hospital Ethics Committee and the participants gave informed consent.

AN was identified and classified according to the following scale: 0 , no $\mathrm{AN} ; 1+$, fine verrucous plaques with or without pigmentation in the neck or axillae; $2+$, coarse verrucous plaque with or without pigmentation in the neck or axillae; $3+$, coarse verrucous plaque with or without pigmentation on the neck or axillae, on the trunk and on one pair of extremities, and $4+$, more severe than the last one, involving more than one pair of extremities or on mucosal membranes (11). One of the authors (L.M.B.A.) plus a second examiner observed all patients.

The women were from Salvador, Bahia, Northeastern Brazil, where there is a mixed race population, mostly black and mulatto of African American ancestry. Their race was determined based on phenotype appearance and skin color according to Krieger et al. (17).

All patients underwent an oral glucose tolerance test (75 g). Plasma glucose was measured by the glucose-oxidase method and serum insulin by radioimmunoassay 0,1 and $2 \mathrm{~h}$ after the glucose load. The patients were classified as diabetic or as having impaired glucose tolerance according to World Health Organization criteria (18).

Serum levels of total cholesterol, HDLcholesterol, and triglycerides were determined with an auto-analyzer (Vitalab, Selectra, Merck KGaA, Darmstadt, Germany), with standard enzymatic procedures.

The insulin area under the curve (AUC) of the oral glucose tolerance test (0-2 h) was calculated by the trapezoidal method. The $\beta$ cell function (HOMA $\beta$ cell) and the insulin resistance (HOMA IR) were evaluated with a homeostasis model of assessment (19):

HOMA $\beta$ cell $=($ insulin $\mu \mathrm{U} / \mathrm{ml} * 20) /$

fasting glucose $(\mathrm{mmol} / \mathrm{l})$ - 3.5

HOMA IR $=[$ insulin $\mu \mathrm{U} / \mathrm{ml} \times$ fasting glucose $(\mathrm{mmol} / \mathrm{l})] / 22.5$ 


\section{Statistical analysis}

Statistical analysis was performed using the "Epi-Info" software, version 6.0. The Student $t$-test was applied to normally distributed data and analysis of variance (MannWhitney and Kruskal-Wallis tests) to other data distributions. For proportion analysis, the Epi-Table calculator was used. The differences were considered to be statistically significant when $\mathrm{P} \leq 0.05$.

Logistic regression was performed using the Statistical Package for the Social Sciences (SPSS, version 9.0). BMI and HOMA insulin resistance were normalized using logarithmic transformation. AN was recorded as presence or absence of the attribute.

The five subgroups of AN were compared with the other variables by ANOVA adjusted for multiple comparison (Bonferroni).

\section{Results}

Clinical characteristics, fasting glucose and insulin levels, insulin AUC and HOMA insulin resistance of the patients according to the degree of AN are presented in Table 1. Age, BMI and waist diameter were similar for the AN and non-AN groups.

The AN group had similar fasting glucose, $B$ cell function, fasting insulin levels and insulin AUC when compared with the non-AN group. Only HOMA insulin resistance was significantly higher in the AN group as compared to the non-AN group $(\mathrm{P}=$ $0.02)$.

Fasting glucose levels among subgroups of AN were similar. Fasting insulin levels were higher in subgroup 4 of AN than in subgroup $1(\mathrm{P}=0.04)$. HOMA insulin resistance was significantly higher in subgroup 4 of AN compared to all other subgroups (Table 1).

Seventy-six percent of the whole group had AN. The race distribution was $35 \%$ white, $38 \%$ mulatto and $27 \%$ black obese women. The frequency of AN in black $(83.8 \%)$ and mulatto $(82.1 \%)$ women was higher than in white women $(62.5 \%)(\mathrm{P}=0.003$ and 0.002 ,

Table 1. Clinical and metabolic parameters for the groups of obese women.

\begin{tabular}{|c|c|c|c|c|c|c|}
\hline & \multicolumn{5}{|c|}{ Grade of acanthosis nigricans } & \multirow[t]{2}{*}{$P$} \\
\hline & $\begin{array}{c}0 \\
(\mathrm{~N}=56)\end{array}$ & $\begin{array}{c}1 \\
(\mathrm{~N}=82)\end{array}$ & $\begin{array}{c}2 \\
(N=69)\end{array}$ & $\begin{array}{c}3 \\
(N=28)\end{array}$ & $\begin{array}{c}4 \\
(N=11)\end{array}$ & \\
\hline Age (years) & $37 \pm 9$ & $35 \pm 9$ & $36 \pm 9$ & $34 \pm 10$ & $34 \pm 9$ & 0.83 \\
\hline BMI $\left(\mathrm{kg} / \mathrm{m}^{2}\right)$ & $38 \pm 8$ & $39 \pm 6$ & $41 \pm 7$ & $42 \pm 6$ & $42 \pm 8$ & 0.12 \\
\hline Waist (cm) & $102 \pm 12$ & $103 \pm 12$ & $106 \pm 12$ & $107 \pm 12$ & $109 \pm 11$ & 0.56 \\
\hline Fasting glucose (mmol/l) & $4.8 \pm 0.8$ & $4.8 \pm 0.9$ & $5.0 \pm 1.0$ & $4.8 \pm 0.6$ & $5.4 \pm 0.9$ & 0.20 \\
\hline Fasting insulin $(\mathrm{pmol} / \mathrm{l})$ & $116 \pm 69$ & $111 \pm 80$ & $114 \pm 70$ & $118 \pm 62$ & $180 \pm 94 *$ & 0.08 \\
\hline $\begin{array}{l}\text { Insulin AUC } \\
\left(\text { pmol l-1 } 0-2 \mathrm{~h}^{-1}\right)\end{array}$ & $396 \pm 240$ & $378 \pm 258$ & $414 \pm 336$ & $438 \pm 222$ & $492 \pm 240$ & 0.65 \\
\hline HOMA IR & $4.1 \pm 2.4$ & $4.2 \pm 3.8$ & $4.3 \pm 2.8$ & $4.2 \pm 2.3$ & $7.5 \pm 4.8^{* * \#}$ & 0.02 \\
\hline HOMA B cell & $494 \pm 327$ & $460 \pm 302$ & $465 \pm 306$ & $489 \pm 266$ & $649 \pm 310$ & 0.40 \\
\hline Total cholesterol $(\mathrm{mmol} / \mathrm{l})$ & $4.8 \pm 1.1$ & $4.8 \pm 0.9$ & $4.8 \pm 1.3$ & $4.5 \pm 1.0$ & $4.9 \pm 1.1$ & 0.62 \\
\hline HDL-cholesterol (mmol/l) & $1.16 \pm 0.33$ & $1.15 \pm 0.36$ & $1.19 \pm 0.26$ & $1.12 \pm 0.31$ & $1.17 \pm 0.30$ & 0.74 \\
\hline Triglycerides (mg\%) & $119 \pm 43$ & $124 \pm 56$ & $114 \pm 52$ & $112 \pm 53$ & $141 \pm 69$ & 0.57 \\
\hline
\end{tabular}

Data are reported as means \pm SD. BMI, body mass index; AUC, area under the curve; HOMA, homeostasis model of assessment; IR, insulin resistance; HDL, high-density lipoprotein.

$* P<0.05$, grade 1 vs grade 4 ;

$* * \mathrm{P}<0.01$, grade 0.1 or 2 vs grade 4 ;

$\#$ P $<0.05$, grade 3 vs grade 4 (ANOVA). 
respectively). Figure 1 shows these data.

AN was 2.5 times (95\% CL: 1.2-5.2) more frequent in the mulatto race than in the white race. This difference increased to 4.1 times (95\% CL: 1.7-9.4) when black women were compared to white women after adjusting for BMI, age and HOMA insulin resistance. BMI and age were significantly associated with $\mathrm{AN}(\mathrm{P}=0.004)$ but HOMA insulin resistance was not $(\mathrm{P}=0.54)$.

The frequencies of diabetes mellitus and impaired glucose tolerance were 5.8 and $12.6 \%$ and tended to be higher in patients with AN than in patients without AN (1.6 and $8.2 \%$, respectively), although the differences were not significant.

The mean total cholesterol, HDL-cholesterol and triglyceride levels were similar for the group with AN and the group without AN. The frequencies of total cholesterol levels $\geq 200 \mathrm{mg} \%$ (28.8 and $27.9 \%$, AN and non-AN group, respectively), HDL-cholesterol levels $<35 \mathrm{mg} \%$ (16.8 and $14.8 \%$ ) or triglyceride levels $\geq 200 \mathrm{mg} \%$ (8.4 and $6.6 \%$ ) were similar.

\section{Discussion}

AN has been considered a marker of insulin resistance and a risk factor for type 2 diabetes mellitus, especially in young adults

Figure 1. Relative frequency (\%) of various grades of acanthosis nigricans according to race.

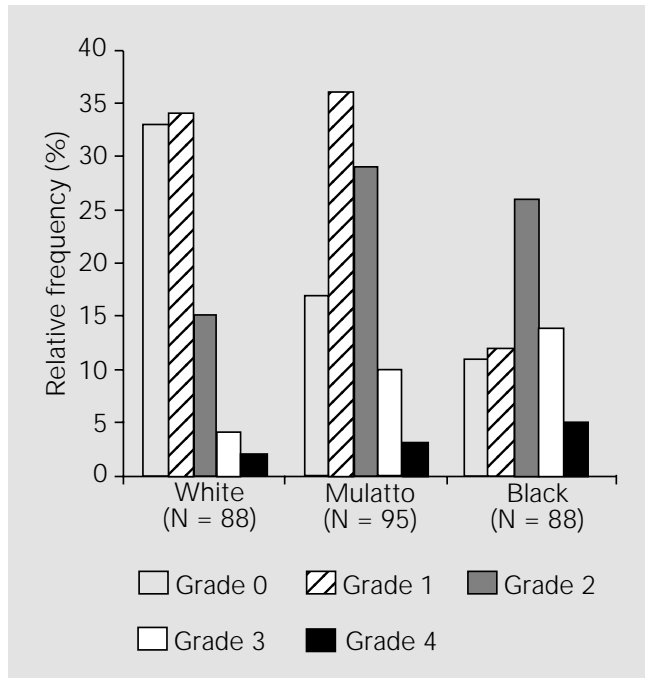

$(2-9,12,14,15)$. In the current study, a stronger association with AN was observed in black and mulatto women than in white obese women of a mixed race population. In contrast, HOMA insulin resistance was not associated with the AN group in multivariate analysis.

Hud et al. (11) have described higher fasting insulin levels in 25 obese women with AN in contrast to 9 without AN but this increase was not significant. Stuart et al. (10) studied fasting insulin levels in 1412 obese children and showed a good correlation between fasting insulin concentration and the severity of AN. Matsuoka et al. (8) observed elevation of fasting glucose in $42 \%$ of 26 patients with endocrine and dermatological diseases and with AN. They also showed increased levels of fasting insulin and most of them had an exaggerated peak of insulin secretion in response to glucose overload. In contrast, another author did not find an increase in insulin response to glucose in women with polycystic ovaries and with $\mathrm{AN}$ versus patients without $A N(7)$.

The present study has also shown higher HOMA insulin resistance in the subgroup with the highest degree of AN compared to the non-AN group or to the other subgroups. Insulin resistance, diabetes mellitus or impaired glucose tolerance, low LDL-cholesterol and high triglycerides are the best markers of metabolic syndromes. In our study, all patients were obese and the frequency of diabetes mellitus (5.8\%) and impaired glucose tolerance $(12.6 \%)$ tended to be higher in the AN group although lipid profiles were similar. In a study on 89 African Americans, Stuart et al. (13) observed non-insulin-dependent diabetes mellitus in $20 \%$ of the subjects with AN. Nearly a two-fold higher prevalence was observed in African Americans compared with US whites. It was suggested that AN would identify a subset with a higher risk to develop non-insulin-dependent diabetes mellitus. Burke et al. (16) have studied 137 Mexican Americans from the 
San Antonio Texas Family Diabetes Study or from the San Antonio Texas Family Heart Study of both sexes compared to 269 individuals without $A N$, all submitted to the oral glucose tolerance test. According to American Diabetes Association criteria, they found type 2 diabetes in $27 \%$ patients of the AN group versus $19.7 \%$ in the non-AN group but the AN group subjects were younger and included many more obese subjects ( 89 versus $52.4 \%$ ) than the non-AN group. They observed no differences in total cholesterol and triglyceride levels between the groups, as also observed in the present study. They also showed that an elevated degree of AN was associated with higher fasting insulin and 2-h glucose after the oral glucose tolerance test, with low HDL-cholesterol and diastolic blood pressure in non-diabetic subjects but with a higher total cholesterol, only in diabetic patients. These higher frequencies of metabolic abnormalities in comparison to our data may be related to ethnic, nutritional or environmental factors.

In the present report, a global prevalence of AN was shown in $76 \%$ of the obese women, similar to the $74 \%$ value reported by Hud et al. (11) whose study included $56 \%$ of black obese women. A higher AN prevalence in black obese women compared with white (85 and $57 \%$, respectively) was also shown. A higher degree of AN in blacks and mulattos was observed. Some authors emphasize the black race as one important determinant factor for AN (12). Stuart et al. (10) have found a much lower frequency of AN (7.1\%) in 1412 obese children of white, black and Hispanic extraction, but only $27 \%$ of them were obese. They observed an AN frequency of $0.5 \%$ in white, $5.7 \%$ in Hispanic and $13.3 \%$ in African American subjects. In 110 African American adults, Stuart et al. (13) observed a $77 \%$ frequency of AN.

Barbiere et al. (3) described increased androgen production, insulin resistance and $\mathrm{AN}$, or hyperandrogenemia insulin resistance-AN syndrome in women with ovarian or adrenal tumors, congenital or adrenal hyperplasia, and gonadal dysgenesis. In these cases, chronic hyperinsulinemia may stimulate the increased ovarian androgen secretion and epidermis proliferation, which result in hirsutism, virilization and AN.

The etiology of AN is probably related to increased levels of a keratinocyte-stimulating factor and of dermal fibroblasts. Insulin at high concentrations may activate insulinlike growth factor receptors and mediate epidermal cell proliferation $(20,21)$. In cases of malignancy and pituitary adenoma associated with $\mathrm{AN}$, an increased production of growth factors, insulin-like activity and transforming growth factor- $\alpha$ have been described (9). The latter is structurally related to epidermal growth factor. Antibodies against insulin receptors could explain the AN association with other autoimmune diseases (22,23).

The presence of AN in this group of obese women was strongly associated with black and mulatto race but weakly with insulin AUC and HOMA insulin resistance, in spite of higher insulin resistance. Since this lesion is easily recognized by skin inspection, it should be carefully monitored in terms of the development of disorders of insulin resistance.

\section{Acknowledgments}

We greatly appreciate the cooperation of Rosa T. Fukui, Carbohydrate Laboratory, Universidade de São Paulo, where the insulin determinations were performed, and the technical assistance of Ivanise Silva and Aldenice Viana de Carvalho, Universidade Federal da Bahia. 


\section{References}

1. Schwartz RA (1994). Acanthosis nigricans. J ournal of the American Academy of Dermatology, 31: 1-19.

2. Kahn CR, Flier J S, Bar RS, Archer JA, Gorden P, Martin MM \& Roth J (1976). The syndrome of insulin resistance and acanthosis nigricans: insulin-receptor disorders in man. New England J ournal of Medicine, 294: 739-745.

3. Barbiere RL, Smith S \& Ryan KJ (1983). Hyperandrogenism, insulin resistance, and acanthosis nigricans syndrome: a common endocrinopathy with distant pathophysiologic features. American J ournal of Obstetrics and Gynecology, 147: 90-99.

4. Flier J S (1985). Metabolic importance of acanthosis nigricans. Archives of Dermatology, 121: 193-194.

5. Peters EJ, Stuart CA \& Prince EJ (1986). Prevalence of acanthosis nigricans and obesity: acquired and intrinsic defects in insulin action. Metabolism: Clinical and Experimental, 35: 807-813.

6. Flier J S, Eastman RC, Minaker KL, Matteson D \& Rowe J W (1987). Acanthosis nigricans in obese women with hyperandrogenism. Characterization of an insulin resistant state distinct of the type $A$ and $B$ syndrome. Diabetes, 34: 101-107.

7. Dunaif A, Graf M, Mandeli J , Laumas V \& Dobrjansky A (1987). Characterization of groups of hyperandrogenic women with acanthosis nigricans, impaired glucose tolerance, and/or hyperinsulinemia. J ournal of Clinical Endocrinology and Metabolism, 65: 499-507.

8. Matsuoka LY, Wortsman J, Gavin J R \& Goldman J (1987). Spectrum of endocrine abnormalities associated with acanthosis nigricans. American J ournal of Medicine, 83: 719-725.

9. Barth J H, Ng LL \& Wojnarowska F (1988). Acanthosis nigricans, insulin resistance and cutaneous virilism. British J ournal of Dermatology, 118: 613-619.

10. Stuart CA, Pate CJ \& Peters EJ (1989). Prevalence of acanthosis nigricans in an unselected population. American J oumal of Medicine, 87: 269-272.

11. Hud J A, Cohen J B, Wagner J M \& Cruz PD (1992). Prevalence and significance of acanthosis nigricans in an adult obese population. Archives of Dermatology, 128: 941-944.

12. Stuart $C A$, Smith $M M$, Gilkison $C R$, Shaheb S \& Stahn RM (1994). Acanthosis nigricans among native Americans: an indicator of high diabetes risk. American J ournal of Public Health, 84: 1839-1842.

13. Stuart CA, Gilkison CR, Keenan BS \& Nagamani M (1997). Hyperinsulinemia and acanthosis nigricans in African Americans. J ournal of the National Medical Association, 89: 523-527.

14. Panidis D, Skiadopoulos S, Rousso D, Ioannides D \& Panidou E (1995). Association of acanthosis nigricans with insulin resistance in patients with polycystic ovary syndrome. British J ournal of Dermatology, 132: 936-941.

15. Stuart CA, Gilkison $C R$, Smith $M M$, Bosma AM, Keenan BS \& Nagamani M (1998). Acanthosis nigricans as a risk factor for non-insulin dependent diabetes mellitus. Clinical Pediatrics, 37: 73-80.

16. Burke JP, Hale DE, Hazuda HP \& Stern MP (1999). A quantitative scale of acanthosis nigricans. Diabetes Care, 22: 16551659.
17. Krieger $\mathrm{H}$, Norton $\mathrm{NE}$, Azevedo $\mathrm{E}$, FreireMaia A \& Yasuda N (1965). Racial admixture in north-eastern Brazil. American J ournal of Human Genetics, 29: 113-125.

18. World Health Organization (1995). Diabetes mellitus: Report of a WHO Study Group. World Health Organization, Teach Report Series, No. 727, Geneva, Switzerland.

19. Matthews DR, Hosker J P, Rudenski AS, Naylor BA, Treacher DF \& Turner RL (1985). Homeostasis model assessment: insulin resistance and B-cell function from fasting plasma glucose and insulin concentrations in man. Diabetologia, 28: 412419.

20. Ellis DL, Kafka SP, Chow J C, Nanney LB, Inman WH, McCadden ME \& King J r LE (1987). Melanoma, growth factors, acanthosis nigricans, the sign of Leser-Trelat, and multiple acronchordons: a possible role for alpha-transforming growth factor in cutaneous paraneoplastic syndromes. New England J oumal of Medicine, 17: 1582-1587.

21. Mirsa P, Nickoloft BJ , M orhenn UB, Hintz RL \& Rosenfeld RG (1986). Characterization of insulin-like growth factor-1/somatomedin $\mathrm{C}$ receptors on human keratinocyte monolayers. J ournal of Investigative Dermatology, 87: 264-267.

22. Moller DE \& Flier J S (1991). Insulin resistance mechanisms, syndromes, and implications. New England J ournal of Medicine, 325: 938-948.

23. Flier J (1992). Lilly lecture: syndromes of insulin resistance from patient to gene and back again. Diabetes, 41: 1207-1219. 